\title{
Some Characterization Results by Conditional Expectations and their Applications to Lindley-type Distributions
}

\author{
M.E. Ghitany ${ }^{1}$, Ramesh C. Gupta ${ }^{2} \&$ Shaochen Wang ${ }^{3}$ \\ ${ }^{1}$ Department of Statistics and Operations Research, Faculty of Science, Kuwait University, Kuwait \\ 2 Department of Mathematics and Statistics, University of Maine, Orono, ME, USA \\ ${ }^{3}$ School of Mathematics, South China University of Technology, Guangzhou, P.R. China \\ Correspondence: M.E. Ghitany, Department of Statistics and Operations Research, Faculty of Science, Kuwait University, \\ Kuwait. E-mail: meghitany@yahoo.com
}

Received: October 7, 2017 Accepted: October 24, 2017 Online Published: November 16, 2017

doi:10.5539/ijsp.v7n1p86 URL: https://doi.org/10.5539/ijsp.v7n1p86

\begin{abstract}
This paper deals with some characterization results based on truncated expectations in the continuous as well as in the discrete case. Both the right and left truncations are considered and some general results are derived. Some of the known results dealing with truncated moments, residual moments and residual partial moments are obtained as special cases. These results are utilized to obtain certain characterization results for the Lindley type distributions. The characterization results provide new methods for estimating the unknown parameter of Lindley-type distributions and their goodness-of-fit. The results on the Lindley-type distributions are applied on some real data sets.
\end{abstract}

Keywords: Lindley distribution, discrete Lindley distribution, conditional expectation, characterization of distributions, Least squares regression line.

\section{Introduction}

Characterizations of probability distributions are important issues in statistical inference. For example, Su and Huang (2000) studied the characterizations of distributions based on conditional expectations. Recently, Nanda (2010) studied the characterizations through mean residual life and failure rates functions of absolutely continuous random variables. Ahmed (1991) characterized beta, binomial and Poisson distributions by connecting conditional expectations with hazard rate functions. Laurent (1974) presented characterization of distributions by truncated moments. Gupta and Gupta (1983) characterized distributions by the moments of residual life.

In this paper, we obtain some general results for the characterization of distributions, both in the continuous as well as in the discrete case. More specifically, we obtain some characterization results knowing $E[g(X) \mid X \geq x]$ and $E[g(X) \mid X \leq x]$. Results are provided to characterize distributions by means of truncated moments, residual moments, reversed residual moments and partial moments. We are particularly interested in the characterizations of continuous and discrete Lindley distributions.

A random variable $X$ is said to have the Lindley distribution if its probability density function (p.d.f.) is given by

$$
f_{L}(x)=\frac{\beta^{2}}{1+\beta}(1+x) e^{-\beta x}, \quad x>0, \quad \beta>0 .
$$

Lindley distribution was first introduced by Lindley (1958) and it is now popular in modeling lifetime data. Recently, Lindley distribution and its extensions have gained much attention in the statistical literature. We will not review this here and refer the reader to Al-Mutairi et al. (2013), Ghitany et al. (2008), (2011), (2013) and the references therein.

Recently, Ahsanullah et al. (2017) provided two characterizations of Lindley distributions based on a relation between left (right) truncated moments and (reversed) failure rate function.

A random variable $X$ is said to have the discrete Lindley distribution if its probability mass function (p.m.f.) is given by

$$
f_{D L}(x)=P(X=x)=\left(1-e^{-\beta}\right)^{2}(1+x) e^{-\beta x}, \quad x=0,1, \ldots, \quad \beta>0 .
$$

Discrete Lindley distribution and its applications are introduced by Mazucheli and de Oliveira (2016). Another discrete Lindley distribution was studied by Gómez-Déniz and Calderín-Ojeda (2011).

In this paper, we give new characterizations of the above continuous/discrete Lindley distributions based on the modified left/right truncated moments. Our new characterizations based on the modified left truncated moments can be used to 
check graphically the goodness-of-fit of the continuous/discrete Lindley distributions. Also, our characterizations provide simple estimates of the parameter in these distributions.

The paper is organized as follows. In section 2, we present some general results, in the continuous case and give some examples. Section 3 contains similar results for the discrete case. Some characterizations of Lindley type distributions are derived in section 4. Two applications are presented in Section 5 to illustrate the importance of the characterization results for Lindley-type distributions.

\section{Characterization Results - Continuous Case}

In this section, we shall assume that $X$ is a continuous random variable with absolutely continuous distribution function $F(x)$, survival function $\bar{F}(x)=1-F(x)$, probability density function $f(x)$, failure rate function $r(x)=f(x) / \bar{F}(x)$ and reversed failure rate function $\tilde{r}(x)=f(x) / F(x)$. It is well known that the failure rate function and the reversed failure rate function characterize the distribution, see for example, Marshall and Olkin (2007).

\subsection{Characterization through Truncated Conditional Expectation}

Let $g(x)$ be a differentiable function such that $0<E[g(X)]<\infty$. Also assume that $g(t) \bar{F}(t) \rightarrow 0$ as $t \rightarrow \infty$. Then

$$
\psi(x)=E[g(X) \mid X \geq x]=\frac{1}{\bar{F}(x)} \int_{x}^{\infty} g(t) f(t) d t=-\frac{1}{\bar{F}(x)} \int_{x}^{\infty} g(t) d \bar{F}(t) .
$$

Integrating the above integral by parts, under the assumed conditions, it can be verified that

$$
\psi(x)=E[g(X) \mid X \geq x]=g(x)+\frac{1}{\bar{F}(x)} \int_{x}^{\infty} g^{\prime}(t) \bar{F}(t) d t .
$$

We shall now obtain an expression for the failure rate in terms of the function $\psi(x)$ and $g(x)$.

The equation (3) can be written as

$$
\bar{F}(x)[\psi(x)-g(x)]=\int_{x}^{\infty} g^{\prime}(t) \bar{F}(t) d t .
$$

Differentiating the above equation and simplifying, we get the failure rate as

$$
r(x)=\frac{\psi^{\prime}(x)}{\psi(x)-g(x)} .
$$

Example 2.1 Characterization through truncated moments

Let $\psi(x)=E\left[X^{k} \mid X \geq x\right\}=m_{k}(x), k=1,2, \ldots$ Then

$$
r(x)=\frac{m_{k}^{\prime}(x)}{m_{k}(x)-x^{k}},
$$

see Navarro and Ruiz (1998).

Example 2.2 Characterization through residual moments

Let $\psi(x)=E\left[(X-x)^{k} \mid X \geq x\right]=h_{k}(x), k=1,2, \ldots$ In this case, we have

$$
h_{k}(x) \bar{F}(x)=-\int_{x}^{\infty}(t-x)^{k} d \bar{F}(t) .
$$

Assuming $t^{k} \bar{F}(t) \rightarrow 0$ as $t \rightarrow \infty$ for all $k$ and integrating by parts, we have

$$
h_{k}(x) \bar{F}(x)=k \int_{0}^{\infty} u^{k-1} \bar{F}(x+u) d u .
$$

Differentiating the above equation and simplifying, we have

$$
r(x)=\frac{h_{k}^{\prime}(x)+k h_{k-1}(x)}{h_{k}(x)}
$$


Thus, in this case, two consecutive residual moments are needed to characterize the distribution.

Example 2.3 Characterization through partial moments

Let $\psi(x)=E\left[(X-x)^{k} \mid X \geq x\right]=g_{k}(x) / \bar{F}(x), k=1,2, \ldots$ In this case

$$
\begin{aligned}
g_{k}(x) & =-\int_{x}^{\infty}(t-x)^{k} d \bar{F}(t) \\
& =-\int_{x}^{\infty}\left[t^{k}-k t^{k-1} x+\left(\begin{array}{l}
k \\
2
\end{array}\right) t^{k-2} x^{2}+\ldots+(-1)^{k} x^{k}\right] d \bar{F}(t) .
\end{aligned}
$$

Differentiating $k$ times with respect to $x$ and simplifying, we get

$$
\bar{F}(x)=(-1)^{k} \frac{g_{k}^{(k)}(x)}{k !} .
$$

Thus, one partial moment is enough to characterize the distribution. For more details, see Gupta and Gupta (1983).

\section{Example 2.4 Characterization through certain truncated conditional expectations}

Let $\psi(x)=E[g(X) \mid X \geq x]=h(x) / \bar{F}(x)$. In this case, using (3), we have

$$
\frac{h(x)}{\bar{F}(x)}=g(x)+\frac{1}{\bar{F}(x)} \int_{x}^{\infty} g^{\prime}(t) \bar{F}(t) d t
$$

This can be written as

$$
h(x)=g(x) \bar{F}(x)+\int_{x}^{\infty} g^{\prime}(t) \bar{F}(t) d t .
$$

Differentiating the above equation, we get

$$
f(x)=-\frac{h^{\prime}(x)}{g(x)}
$$

\subsection{Characterization by Reversed Truncated Conditional Expectation}

Let $g(x)$ be a differentiable function such that $0<E[g(X)]<\infty$. Also assume that $g(0)$ is finite and $g(0) F(0)=0$. Then

$$
\phi(x)=E[g(X) \mid X \leq x]=\frac{1}{F(x)} \int_{0}^{x} g(t) f(t) d t=\frac{1}{F(x)} \int_{0}^{x} g(t) d F(t) .
$$

Integrating the above integral by parts, under the assumed conditions, it can be verified that

$$
\phi(x)=E[g(X) \mid X \leq x]=g(x)-\frac{1}{F(x)} \int_{0}^{x} g^{\prime}(t) F(t) d t .
$$

We shall now obtain an expression for the reversed failure rate in terms of the function $\phi(x)$ and $g(x)$.

The quation (9) can be written as

$$
F(x)[\phi(x)-g(x)]=-\int_{0}^{x} g^{\prime}(t) F(t) d t .
$$

Differentiating the above equation and simplifying, we get the reversed failure rate as

$$
\tilde{r}(x)=\frac{\phi^{\prime}(x)}{g(x)-\phi(x)} .
$$

Example 2.1R Characterization through reversed truncated moments

Let $\phi(x)=E\left[X^{k} \mid X \leq x\right\}=M_{k}(x), k=1,2, \ldots$ Then

$$
\tilde{r}(x)=\frac{M_{k}^{\prime}(x)}{x^{k}-M_{k}(x)},
$$


Example 2.2R Characterization through reversed residual moments

Let $\phi(x)=E\left[(X-x)^{k} \mid X \leq x\right]=H_{k}(x), k=1,2, \ldots$ In this case, we have

$$
H_{k}(x) F(x)=\int_{-\infty}^{x}(t-x)^{k} d F(t) .
$$

Assuming $t^{k} F(t) \rightarrow 0$ as $t \rightarrow-\infty$ for all $k$ and integrating by parts, it can be verified that

$$
H_{k}(x) F(x)=k \int_{-\infty}^{0} u^{k-1} F(x+u) d u .
$$

Differentiating the above equation and simplifying, it can be verified that

$$
\tilde{r}(x)=-\frac{H_{k}^{\prime}(x)+k H_{k-1}(x)}{H_{k}(x)}
$$

Thus, in this case, two reversed consecutive residual moments are needed to characterize the distribution.

Example 2.3R Characterization through partial reversed moments

Let $\phi(x)=E\left[(X-x)^{k} \mid X \leq x\right]=G_{k}(x) / F(x), k=1,2, \ldots$ In this case

$$
\begin{aligned}
G_{k}(x) & =\int_{0}^{x}(t-x)^{k} d F(t) \\
& =\int_{0}^{x}\left[t^{k}-k t^{k-1} x+\left(\begin{array}{l}
k \\
2
\end{array}\right) t^{k-2} x^{2}+\ldots+(-1)^{k} x^{k}\right] d F(t) .
\end{aligned}
$$

Differentiating $k$ times with respect to $x$ and simplifying, it can be verified that

$$
F(x)=(-1)^{k} \frac{G_{k}^{(k)}(x)}{k !} .
$$

Thus, one partial reversed moment is enough to characterize the distribution. For more details, see Gupta and Gupta (1983).

Example 2.4R Characterization through certain reversed truncated conditional expectations

Let $\phi(x)=E[g(X) \mid X \leq x]=H(x) / F(x)$. In this case, using (9), we have

$$
\frac{H(x)}{F(x)}=g(x)-\frac{1}{F(x)} \int_{0}^{x} g^{\prime}(t) F(t) d t
$$

This can be written as

$$
H(x)=g(x) F(x)-\int_{0}^{x} g^{\prime}(t) F(t) d t .
$$

Differentiating the above equation, we get

$$
f(x)=\frac{H^{\prime}(x)}{g(x)}
$$

\section{Characterization Results - Discrete Case}

In this section, we shall assume that $X$ is a discrete random variable defined on the non-negative integers with probability mass function $P(X=x)$ and survival function $\bar{F}(x)=P(X \geq x)$. 


\subsection{Characterization through Truncated Conditional Expectation}

In this case

$$
\begin{aligned}
\psi(x) & =E[g(X) \mid X \geq x] \\
& =\frac{1}{\bar{F}(x)} \sum_{i=x}^{\infty} g(i) P(X=i) \\
& =\frac{1}{\bar{F}(x)} \sum_{i=x}^{\infty} g(i)[\bar{F}(i)-\bar{F}(i+1)] \\
& =g(x)+\frac{1}{\bar{F}(x)} \sum_{i=x}^{\infty}[g(i+1)-g(i)] \bar{F}(i+1) .
\end{aligned}
$$

The above expression can also be written as

$$
[\psi(x)-g(x)] \bar{F}(x)=\sum_{i=x}^{\infty}[g(i+1)-g(i)] \bar{F}(i+1) .
$$

Since the above result is true for all $x$, we change $x$ to $x+1$ and subtract to obtain

$$
\frac{\bar{F}(x+1)}{\bar{F}(x)}=\frac{\psi(x)-g(x)}{\psi(x+1)-g(x)} .
$$

This gives

$$
\bar{F}(x)=F(0) \prod_{j=0}^{x-1} \frac{\psi(j)-g(j)}{\psi(j+1)-g(j)} .
$$

Now

$$
\begin{aligned}
P(X=x) & =\bar{F}(x)-\bar{F}(x+1) \\
& =\bar{F}(x)\left[\frac{\psi(x)-\psi(x+1)}{g(x)-\psi(x+1)}\right],
\end{aligned}
$$

where $\bar{F}(x)$ is given by (16).

Remark 1. Gupta (1985) have characterized a distribution distribution by means of two consecutive factorial moments.

The failure rate function is given by

$$
r(x)=\frac{P(X=x)}{\bar{F}(x)}=\frac{\psi(x)-\psi(x+1)}{g(x)-\psi(x+1)} .
$$

Remark 2. A similar result was obtained by Dimaki and Xekalaki (1996). However, there is an error in their result.

\section{Example 3.1 Characterization through truncated moments}

Let $\psi(x)=E\left(X^{k} \mid X \geq x\right)=m_{k}(x), k=1,2, \ldots$ Then, using (18), we have

$$
r(x)=\frac{m_{k}(x+1)-m_{k}(x)}{m_{k}(x+1)-x^{k}}
$$

\section{Example 3.2 Characterization through residual moments}

Let $\psi(x)=E\left[(X-x)^{k} \mid X \geq x\right]=h_{k}(x), k=1,2, \ldots$ In this case, we have

$$
r(x)=\frac{h_{k}(x+1)-h_{k}(x)}{h_{k}(x+1)} .
$$

This means that the $k$ th residual moment at two consecutive points is needed to characterize a distribution. 
Example 3.3 Characterization through certain truncated conditional expectations

We shall now consider the case when $\psi(x)=E[g(X) \mid X \geq x]=h(x) / \bar{F}(x)$.

(a) Assume $g(x)=0$. In this case, we have

$$
h(x)=\sum_{i=x+1}^{\infty} g(i) P(X=i) .
$$

Therefore, for all $k=1,2, \ldots$,

$$
h(x+k-1)-h(x+k)=g(x+k) P(X=x+k) .
$$

That is, for all $k=1,2, \ldots$,

$$
P(X=x+k)=\frac{h(x+k-1)-h(x+k)}{g(x+k)} .
$$

(b) Assume $g(x) \neq 0$. In this case, we have

$$
h(x)=\sum_{i=x}^{\infty} g(i) P(X=i)
$$

Therefore,

$$
h(x)-h(x+1)=g(x) P(X=x)
$$

That is,

$$
P(X=x)=\frac{h(x)-h(x+1)}{g(x)} .
$$

\section{Example 3.4 Characterization through partial moments}

Let $\psi(x)=E\left[(X-x)^{k} \mid X \geq x\right]=g_{k}(x) / \bar{F}(x), k=1,2, \ldots$ In this case, $g(t)=(t-x)^{k}$ and $g(x)=0$. Therefore, using equation (21), for all $m=1,2, \ldots$, we have

$$
P(X=x+m)=\frac{g_{k}(x+m-1)-g_{k}(x+m)}{m^{k}} .
$$

\subsection{Characterization through Reversed Truncated Conditional Expectation}

In this case

$$
\begin{aligned}
\phi(x) & =E[g(X) \mid X \leq x] \\
& =\frac{1}{F(x)} \sum_{i=0}^{x} g(i) P(X=i) \\
& =\frac{1}{F(x)} \sum_{i=0}^{x} g(i)[F(i)-F(i-1)] \\
& =g(x)+\frac{1}{F(x)} \sum_{i=0}^{x-1}[g(i)-g(i+1)] F(i) .
\end{aligned}
$$

The above expression can also be written as

$$
[\phi(x)-g(x)] F(x)=\sum_{i=0}^{x-1}[g(i)-g(i+1)] F(i) .
$$

Since the above result is true for all $x$, we change $x$ to $x-1$ and subtract to obtain

$$
\frac{F(x-1)}{F(x)}=\frac{\phi(x)-g(x)}{\phi(x-1)-g(x)} \text {. }
$$

This gives

$$
F(x)=F(0) \prod_{j=1}^{x} \frac{\phi(j-1)-\phi(j)}{\phi(j-1)-g(j)} .
$$


Now

$$
\begin{aligned}
P(X=x) & =F(x)-F(x-1) \\
& =F(x)\left[\frac{\phi(x-1)-\phi(x)}{\phi(x-1)-g(x)}\right]
\end{aligned}
$$

The reversed hazard rate function is given by

$$
\tilde{r}(x)=\frac{P(X=x)}{F(x)}=\frac{\phi(x-1)-\phi(x)}{\phi(x-1)-g(x)} .
$$

Example 3.1R Characterization through reversed truncated moments

Let $\phi(x)=E\left[X^{k} \mid X \leq x\right\}=M_{k}(x), k=1,2, \ldots$ Then

$$
\tilde{r}(x)=\frac{M_{k}(x-1)-M_{k}(x)}{M_{k}(x-1)-x^{k}} .
$$

Example 3.2R Characterization through reversed residual moments

Let $\psi(x)=E\left[(X-x)^{k} \mid X \leq x\right]=H_{k}(x), k=1,2, \ldots$ In this case, we have

$$
\tilde{r}(x)=\frac{H_{k}(x-1)-H_{k}(x)}{H_{k}(x-1)} .
$$

Example 3.3R Characterization through certain reversed truncated conditional expectations

We shall now consider the case when $\phi(x)=E[g(X) \mid X \leq x]=H(x) / F(x)$.

(a) Assume $g(x)=0$. In this case, we have

$$
H(x)=\sum_{i=0}^{x-1} g(i) P(X=i) .
$$

Therefore, for all $k=1,2, \ldots, x-1$

$$
H(x-k+1)-H(x-k)=g(x-k) P(X=x-k) .
$$

That is,

$$
P(X=x-k)=\frac{H(x-k+1)-H(x-k)}{g(x-k)} .
$$

(b) Assume $g(x) \neq 0$. In this case, we have

$$
H(x)=\sum_{i=0}^{x} g(i) P(X=i)
$$

Therefore,

$$
H(x)-H(x-1)=g(x) P(X=x) .
$$

That is,

$$
P(X=x)=\frac{H(x)-H(x-1)}{g(x)} .
$$

Example 3.4R Characterization through reversed partial moments

Let $\phi(x)=E\left[(X-x)^{k} \mid X \leq x\right]=G_{k}(x) / \bar{F}(x), k=1,2, \ldots$ In this case, $g(s)=(s-x)^{r}$ and $g(x)=0$. Therefore, using equation (29), for all $m=1,2, \ldots, x-1$, we have

$$
P(X=x-m)=\frac{G_{k}(x-m+1)-G_{k}(x-m)}{g(x-m)} .
$$




\section{Characterization of Lindley Type Distributions}

\subsection{Characterization of Continuous Lindley Distribution}

(i) Characterization through left truncated moments

Theorem 4.1.1 A continuous random variable $X$, with finite $E\left(X^{k}\right)$, follows the Lindley distribution with parameter $\beta$ if and only if, for all $k=1,2, \ldots$,

$$
\bar{F}(x) E\left[X^{k} \mid X \geq x\right]=\frac{\beta^{2}}{1+\beta} \sum_{i=0}^{k+1} c_{n} x^{i} e^{-\beta x}, \quad x>0,
$$

where

$$
c_{0}=\frac{k !(k+1+\beta)}{\beta^{k+2}}, \quad c_{i+1}=\frac{\beta}{i+1} c_{i}, \quad i=0,1, \ldots, k-1, \quad c_{k+1}=\frac{1}{\beta} .
$$

This characterization result can be obtained by choosing

$$
h(x)=\frac{\beta^{2}}{1+\beta} \sum_{i=0}^{k+1} c_{i} x^{i} e^{-\beta x}, \quad g(x)=x^{k} .
$$

Thus, using equation (8), we have

$$
f(x)=-\frac{h^{\prime}(x)}{g(x)}=\frac{\beta^{2}}{1+\beta}(1+x) e^{-\beta x}
$$

Theorem 4.1.1 is equivalent to

$$
E\left[X^{k} \mid X \geq x\right]=\frac{\sum_{i=0}^{k+1} c_{i} x^{i}}{1+x} r(x)
$$

which is the result obtained by Ahsanullah et al. (2017), p. 6224.

(ii) Characterization through modified left truncated moments

Theorem 4.1.2 A continuous random variable $X$ has Lindley distribution with parameter $\beta$ if and only if

$$
\bar{F}(x) E\left[\frac{1}{1+X} \mid X \geq x\right]=\frac{\beta}{1+\beta} e^{-\beta x}, \quad x>0 .
$$

This characterization result can be obtained by choosing

$$
h(x)=\frac{\beta}{1+\beta} e^{-\beta x}, \quad g(x)=\frac{1}{1+x} .
$$

Thus, using equation (8), we have

$$
f(x)=-\frac{h^{\prime}(x)}{g(x)}=\frac{\beta^{2}}{1+\beta}(1+x) e^{-\beta x}
$$

We shall show the importance of this characterization result in the applications section.

(iii) Characterization through right truncated moments

Theorem 4.1.1R A continuous random variable $X$, with finite $E\left(X^{k}\right)$, follows the Lindley distribution with parameter $\beta$ if and only if, for all $k=1,2, \ldots$,

$$
F(x) E\left[X^{k} \mid X \leq x\right]=\frac{\beta^{2}}{1+\beta}\left(c_{0}-\sum_{i=0}^{k+1} c_{i} x^{i} e^{-\beta x}\right), \quad x>0,
$$

where

$$
c_{0}=\frac{k !(k+1+\beta)}{\beta^{k+2}}, \quad c_{i+1}=\frac{\beta}{i+1} c_{i}, \quad i=0,1, \ldots, k-1, \quad c_{k+1}=\frac{1}{\beta} .
$$


This characterization result can be obtained by choosing

$$
H(x)=\frac{\beta^{2}}{1+\beta}\left(c_{0}-\sum_{i=0}^{k+1} c_{i} x^{i} e^{-\beta x}\right), \quad g(x)=x^{k} .
$$

Thus, using equation (14), we have

$$
f(x)=\frac{H^{\prime}(x)}{g(x)}=\frac{\beta^{2}}{1+\beta}(1+x) e^{-\beta x} .
$$

Theorem 4.1.1R is equivalent to is equivalent to

$$
E\left[X^{k} \mid X \leq x\right]=\frac{c_{0} e^{\beta}-\sum_{n=0}^{n+1} c_{n} x^{k}}{1+x} \tilde{r}(x),
$$

which is the result obtained by Ahsanullah et al. (2017), p. 6226.

(vi) Characterization through modified right truncated moments

Theorem 4.1.2R A continuous random variable $X$ has Lindley distribution with parameter $\beta$ if and only if

$$
F(x) E\left[\frac{1}{1+X} \mid X \leq x\right]=\frac{\beta}{1+\beta}\left(1-e^{-\beta x}\right), \quad x>0 .
$$

This characterization result can be obtained by choosing

$$
H(x)=\frac{\beta}{1+\beta}\left(1-e^{-\beta x}\right), \quad g(x)=\frac{1}{1+x} .
$$

Thus, using equation (14), we have

$$
f(x)=\frac{H^{\prime}(x)}{g(x)}=\frac{\beta^{2}}{1+\beta}(1+x) e^{-\beta x} .
$$

\subsection{Characterization of Discrete Lindley Distribution}

(i) Characterization through left truncated moments

Theorem 4.2.1 A discrete random variable $X$ follows the discrete Lindley distribution with parameter $\beta$ if and only if, for all $k=1,2, \ldots$,

$$
\bar{F}(x) E\left[X^{k} \mid X \geq x\right]=\left(1-e^{-\beta}\right)^{2} \sum_{i=x}^{\infty} i^{k}(1+i) e^{-\beta i}, \quad x=0,1, \ldots
$$

This characterization result can be obtained by choosing

$$
h(x)=\left(1-e^{-\beta}\right)^{2} \sum_{i=x+1}^{\infty} i^{k}(1+i) e^{-\beta i}, \quad g(x)=x^{k} .
$$

Note that $g(0)=0$, for all $k=1,2, \ldots$ Now, using equation (21) when $x=0$, for all $m=1,2, \ldots$, we have

$$
P(X=m)=\frac{h(m-1)-h(m)}{g(m)}=\left(1-e^{-\beta}\right)^{2}(1+m) e^{-\beta m},
$$

and

$$
P(X=0)=1-\sum_{m=1}^{\infty} P(X=m)=1-\left(2-e^{-\beta}\right) e^{-\beta}=\left(1-e^{-\beta}\right)^{2} .
$$

(ii) Characterization through modified left truncated moments

Theorem 4.2.2 A discrete random variable $X$ has discrete Lindley distribution with parameter $\beta$ if and only if

$$
\bar{F}(x) E\left[\frac{1}{1+X} \mid X \geq x\right]=\left(1-e^{-\beta}\right) e^{-\beta x}, \quad x=0,1,2, \ldots
$$


In this case $h(x)=\left(1-e^{-\beta}\right) e^{-\beta x}$ and $g(x)=\frac{1}{1+x} \neq 0$. Thus, using equation (22), we have

$$
P(X=x)=\frac{h(x)-h(x+1)}{g(x)}=\left(1-e^{-\beta}\right)^{2}(1+x) e^{-\beta x} .
$$

We shall show the importance of this characterization result in the applications section.

(iii) Characterization through right truncated moments

Theorem 4.2.1R A discrete random variable $X$ follows the discrete Lindley distribution with parameter $\beta$ if and only if, for all $x=0,1, \ldots$ and $k=1,2, \ldots$,

$$
F(x) E\left[X^{k} \mid X \leq x\right]=\left(1-e^{-\beta}\right)^{2} \sum_{i=0}^{x} i^{k}(1+i) e^{-\beta i} .
$$

This characterization result can be easily obtained by choosing

$$
H(x)=\left(1-e^{-\beta}\right)^{2} \sum_{i=0}^{x} i^{k}(1+i) e^{-\beta i}, \quad g(x)=x^{k} .
$$

Here, $g(0)=0$, for all $k=1,2, \ldots$ Thus, using (29), we have $P(X=0)=H(0)=\left(1-e^{-\beta}\right)^{2}$ and

$$
P(X=x)=\frac{H(x)-H(x-1)}{g(x)}=\left(1-e^{-\beta}\right)^{2}(1+x) e^{-\beta x}, \quad x=1,2, \ldots
$$

(vi) Characterization through modified right truncated moments

Theorem 4.2.2R A discrete random variable $X$ follows the discrete Lindley distribution with parameter $\beta$ if and only if

$$
F(x) E\left[\frac{1}{1+X} \mid X \leq x\right]=\left(1-e^{-\beta}\right)\left(1-e^{-\beta(1+x)}\right), \quad x=0,1,2, \ldots
$$

In this case $H(x)=\left(1-e^{-\beta}\right)\left(1-e^{-\beta(1+x)}\right)$ and $g(x)=\frac{1}{1+x} \neq 0$. Thus, using (30), we have

$$
P(X=x)=\frac{H(x)-H(x-1)}{g(x)}=\left(1-e^{-\beta}\right)^{2}(1+x) e^{-\beta x} .
$$

\section{Applications}

\subsection{Application of Characterization of Lindley Distribution}

It has been shown in Theorem 4.1.2 that $X$ follows the Lindley distribution with parameter $\beta$ if and only if

$$
\log \left\{\bar{F}(x) \mathbb{E}\left[\frac{1}{1+X} \mid X \geq x\right]\right\}=\log \left(\frac{\beta}{1+\beta}\right)-\beta x, \quad \text { for all } x>0 .
$$

A natural nonparametric estimator of the left hand side of (32) is

$$
Y(x)=\log \left\{\frac{1}{n} \sum_{i=1}^{n} \frac{1}{1+X_{i}} I\left(X_{i} \geq x\right)\right\}, \quad \text { for all } x>0,
$$

where $X_{1}, \ldots, X_{n}$ is a random sample from the Lindley distribution and $I(A)$ is the indicator function of the set $A$.

Now, based on the data $\left\{\left(x_{i}, Y\left(x_{i}\right)\right), i=1,2, \ldots, n\right\}$, we can find the least squares regression line of the response $Y(x)$ in terms of the predictor $x$. Using this least squares regression line, we can estimate the parameter $\beta$ of the Lindley distribution.

Data set 1: (Lawless 2003, p. 204) This data set represents the failure times (in minutes) for a sample of 15 electronic components in an accelerated life test:

$x=\{1.4,5.1,6.3,10.8,12.1,18.5,19.7,22.2,23,30.6,37.3,46.3,53.9,59.8,66.2\}$. 
For this data set, we have

$y(x)=\{-2.53,-2.96,-3.20,-3.45,-3.65,-3.87,-4.05,-4.25,-4.48,-4.76,-5.04,-5.36,-5.71,-6.17,-6.92\}$.

The least-squares regression line of $y$ on $x$ is given by $\hat{y}(x)=-2.80-0.06 x$. The coefficient of determination $R^{2}$ of this regression line is $97.7 \%$, indicating excellent fit.

Now, using the slope of this regression line, we have $\hat{\beta}=0.06$ Also, using the intercept of this regression line, we have $\log \left(\frac{\hat{\beta}}{1+\hat{\beta}}\right)=-2.80$ which implies $\hat{\beta}=0.06$.

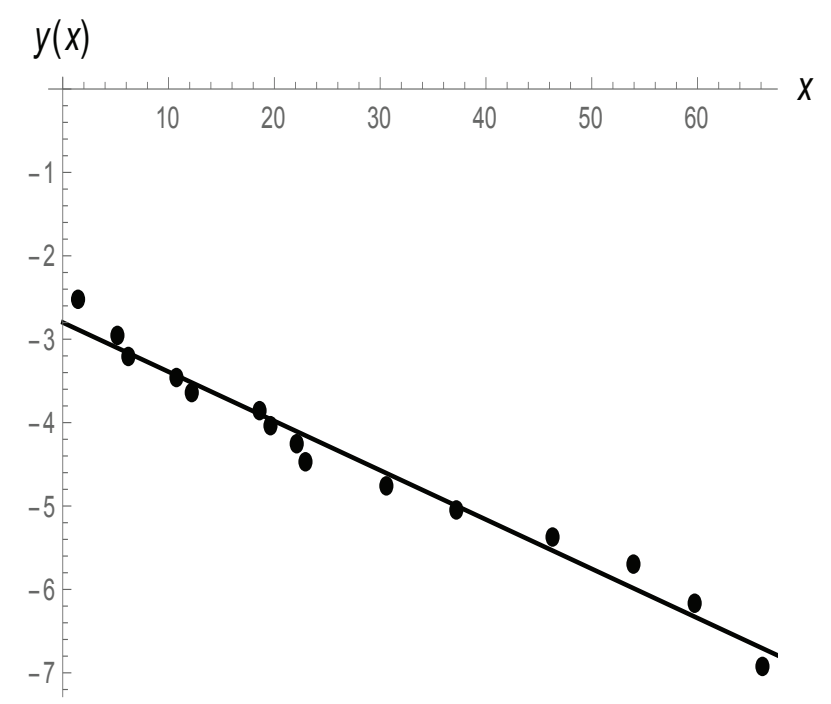

Figure 1. Least squares regression line of $y(x)$ on $x$ of Data set 1.

\subsection{Application of Characterization of Discrete Lindley Distribution}

It has been shown in Theorem 4.2.2 that $X$ follows the discrete Lindley distribution with parameter $\beta$ if and only if

$$
\log \left\{\bar{F}(x) \mathbb{E}\left[\frac{1}{1+X} \mid X \geq x\right]\right\}=\log \left(1-e^{-\beta}\right)-\beta x, \quad \text { for all } x=0,1, \ldots
$$

A natural nonparametric estimator of the left hand side of (34) is

$$
Z(x)=\log \left\{\frac{1}{n} \sum_{i=1}^{n} \frac{1}{1+X_{i}} I\left(X_{i} \geq x\right)\right\}, \quad \text { for all } x=0,1, \ldots,
$$

where $X_{1}, \ldots, X_{n}$ is a random sample from the discrete Lindley distribution and $I(A)$ is the indicator function of the set $A$. Now, based on the data $\left\{\left(x_{i}, Z\left(x_{i}\right)\right), i=1,2, \ldots, n\right\}$, we can find the least squares regression line of the response $Z(x)$ in terms of the predictor $x$. Using this least squares regression line, we can estimate the parameter $\beta$ of the discrete Lindley distribution.

Data set 2: (Falls et al. 1971, p. 98) This data set represents the number of thunderstorm events per day during the month of June at Cape Kennedy, Florida:

$x=\{0(187), 1(77), 2(40), 3(17), 4(6), 5(2), 6(1)\}$, where the numbers between parenthesis are the frequencies.

For this data set, we have

$z(x)=\{-0.30(187),-1.74(77),-2.84(40),-4.02(17),-5.28(6),-6.54(2),-7.75(1)\}$.

The least-squares regression line of $z$ on $x$ is given by $\hat{z}(x)=-0.34-1.26 x$. The coefficient of determination $R^{2}$ of this regression line is $99.7 \%$, indicating excellent fit.

Therefore, using the slope of this regression line, we have $\hat{\beta}=1.26$ Also, using the intercept of this regression line, we have $\log \left(1-e^{-\hat{\beta}}\right)=-0.34$ which implies $\hat{\beta}=1.24$. 


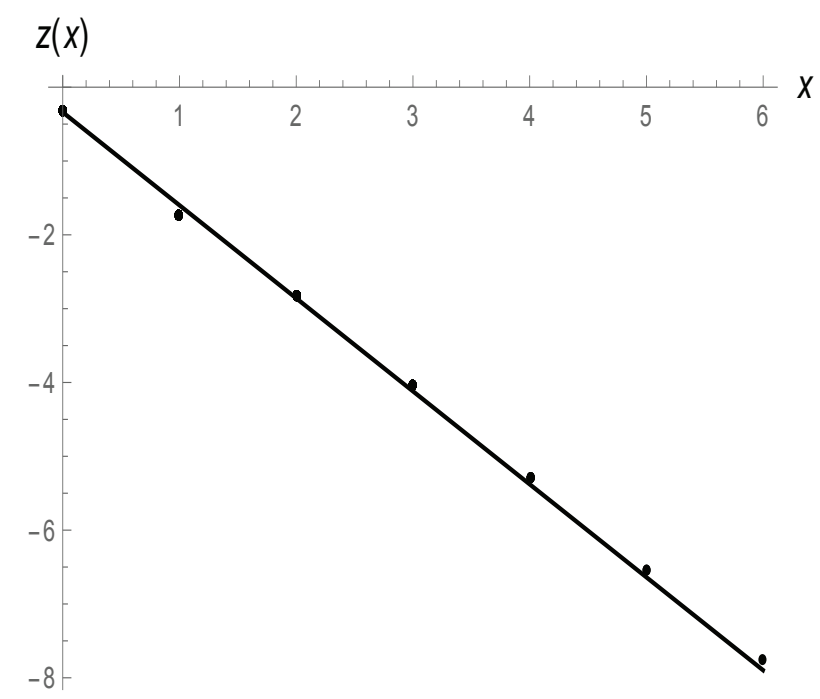

Figure 2. Least squares regression line of $z(x)$ on $x$ of Data set 2.

\section{Conclusions}

In this paper, we have presented some general results based on the conditional expectations, both in the continuous as well as in the discrete case. Some of the ingredients, in the continuous case, are available in the literature. We unify these results dealing with the truncated moments, residual moments and residual partial moments. In addition, similar results are obtained in the case of reversed conditional expectations. The characterization results, in the discrete case, are also derived, both in the case of right truncation as well as in the case of left truncation. The characterization results provide new methods for estimating the unknown parameter of Lindley-type distributions and their goodness-of-fit. Some applications to real data sets are provided in the case of Lindley-type distributions.

\section{References}

Ahmed, A. N. (1991). Characterizations of beta, binomial and Poisson distributions. IEEE Transactions on Reliability, 40(3), 290-295. https://doi.org/10.1109/24.85443

Ahsanullah, M., Ghitany, M. E., \& Al-Mutairi, D. K. (2017). Characterization of Lindley distribution by truncated moments. Communications in Statistics-Theory and Methods, 46(2), 6222-6227. https://doi.org/10.1080/03610926.2015.1124117

Al-Mutairi, D. K., Ghitany, M. E., \& Kundu, D. (2013). Inferences on stress-strength reliability from lindley distributions. Communications in Statistics-Theory and Methods, 42(8), 1443-1463. https://doi.org/10.1080/03610926.2011.563011

Dimaki, C., \& Xekalaki, E. (1996). Towards a unification of certain characterizations by conditional expectations. Annals of the Institute of Statistical Mathematics, 48(1), 157-168. https://doi.org/10.1007/BF00049296

Falls, L. W., Williford, W. O., \& Carter, M. C. (1971). Probability Distributions for thunderstorm activity at Cape Kennedy, Florida. Journal of Applied Meteorology, 10, 97-104. https://doi.org/10.1175/1520-0450(1971)010<0097:PDFTAA > 2.0.CO;2

Galambos, J., \& Kotz, S. (1978). Characterizations of Probability Distributions. Lecture Notes in Mathematics, 675, Springer-Verlag, Berlin, Germany. https://doi.org/10.1007/BFb0069530

Ghitany, M. E., Atieh, B., \& Nadarajah, S. (2008). Lindley distribution and its application. Mathematics and Computers in Simulation, 78, 493-506. https://doi.org/10.1016/j.matcom.2007.11.021

Ghitany, M. E., Alqallaf, F., Al-Mutairi, D. K., \& Husain, H. A. (2011). A two-parameter weighted Lindley distribution and its applications to survival data. Mathematics and Computers in Simulation, 81, 1190-1201. https://doi.org/10.1016/j.matcom.2010.11.005

Ghitany, M. E., Al-Mutairi, D. K., Balakrishnan, N., \& Al-Enezi, L. J. (2013). Power Lindley distribution and associated inference. Computational Statistics and Data Analysis, 64, 20-33. https://doi.org/10.1016/j.csda.2013.02.026 
Gómez-Déniz, E., \& Calderín-Ojeda, E. (2011). The discrete Lindley distribution: properties and applications. Journal of Statistical Computation and Simulation, 81(11), 1405-1416. https://doi.org/10.1080/00949655.2010.487825

Gupta, P. L. (1985). Some characterizations of distributions by conditional moments. Statistics and Probability Letters, 16, 465-473. https://doi.org/10.1080/02331888508801876

Gupta, P. L., \& Gupta, R. C. (1983). On the moments of residual life in reliability and some characterization results. Communications in Statistics-Theory and Methods, 12(4), 449-461. https://doi.org/10.1080/03610928308828471

Lawless, J. F. (2003). Statistical Models and Methods for Lifetime Data, 2nd ed., John Wiley and Sons, New York.

Laurent, A. G. (1974). On characterization of some distributions by truncation properties. Journal of the American Statistical Association, 59, 823-827. https://doi.org/10.1080/01621459.1974.10480213

Lindley, D. V. (1958). Fiducial distributions and Bayess theorem. Journal of the Royal Statistical Society: Series B, 20(1), 102-107.

Marshall, A. W., \& Olkin, I. (2007). Life Distributions, Springer Verlag, Ne York.

Mazucheli, J., \& de Oliveira, R.P. (2016). A Comparative study between two discrete Lindley distributions. Personal communication.

Nanda, A. K. (2010). Characterizations of distributions through failure rate and men residual life function. Statistics and Probability Letters, 80, 752-755. https://doi.org/10.1016/j.spl.2010.01.006

Navarro, J., \& Ruiz, J. M. (1998). Characterizations through moments of the residual life through conditional spacings. Sankhya, Series A, 60, 36-48.

Su, J-C., \& Huang, W-J. (2000). Characterizations based on conditional expectations. Statistical Papers, 41, 423-435. https://doi.org/10.1007/BF02925761

\section{Copyrights}

Copyright for this article is retained by the author(s), with first publication rights granted to the journal.

This is an open-access article distributed under the terms and conditions of the Creative Commons Attribution license (http://creativecommons.org/licenses/by/4.0/). 